\title{
Protein analysis: progress of analytical techniques
}

\author{
Ojha M.D. ${ }^{1}$, Bhandari P.A. ${ }^{2}$, Patil A.G. ${ }^{3}$, Prasade A.M. ${ }^{4}$ \\ ${ }^{1}$ I.C.L.E'S Motilal Jhunjhunwala College of Arts, Science and Commerce, Secytor-9, Vashi, Navi Mumbai \\ ${ }^{2}$ P.T.V.A's Sathaye College, Vile Parle (E), Mumbai-400 057, India \\ ${ }^{3}$ V.E.S., College of Arts, Science and Commerce, Chembur, Mumbai-400 071, India \\ ${ }^{4}$ Gogate Jogalekar College of Science, Arts and Commerce, Ratnagiri, India
}

\begin{abstract}
Protein analysis employs various techniques and methods, which help to study the protein. Quality Control (QC) is a system of routine technical activities, to measure and control the quality of the inventory as it is being developed. It provides routine and consistent checks to ensure data integrity, correctness, and completeness, helps to identify and address errors and omissions also document and archive inventory material and record all QC activities. Protein analysis techniques are developing fast due to the growing number of proteins obtained by recombinant DNA techniques.
\end{abstract}

Keywords- Analytical techniques, DNA Chip, HPLC, LC-MS, GMP, BLAST

\section{Analytical techniques}

There are various number of modifications made in the existing techniques and instruments for more desirable analysis of proteins like, two LCESI-MS and CID-MS/MS methods been developed and validated for the determination of the novel oral 1,14 substituted taxane derivatives, IDN 5738 and IDN 5839, in mouse plasma. Sunfire C18 column was used for HPLC separation and the recovery was more than $90 \%$. Using these methods for the first time the pharmacokinetics of the two taxanes was analyzed [1, 2]. HPLC has found immense application in pharmacokinetics. HPLC has been developed and validated for rapid determination of sinafloxacin, a novel fluoroquinolone in rat plasma using a fused-core C18-silica column. The total analysis time was as short as $3 \mathrm{~min}$. The method was sensitive with a limit of detection (LOD) of $2 \mathrm{ng} \mathrm{ml}-1$, with good linearity [3]. Liquid chromatography-mass spectrometry (LC-APClMS) has been used to analysed predominant triterpenes (free oleanolic acid, $\beta$-sitosterol and $\beta$ sitosterol-3-O- $\beta$-d-glucoside) in skin and cuticular wax of grape berries. The method showed good repeatibility. The grape varities which showed high variability was based triterpene content, quantitites of oleanolic acid of cuticular wax of the twelve analysed samples. The highest sitosterol and sitosterol glucoside content was also found in 'Othello' variety. This study on triterpenes could be an informative tool to characterize grape varieties [4]. Laser ablation inductively coupled plasma mass spectrometry (LA-ICP-MS) using double-focusing sector field (LA-ICP-SFMS) or quadrupole-based mass spectrometers (LA-ICPQMS) has been successfully applied as a powerful imaging (mapping) technique to produce quantitative images of detailed regionally specific element distributions in thin tissue sections of human or rodent brain. It was also applied to investigate metal distributions in plant and animal sections to study, the uptake and transport of nutrient and toxic elements or environmental contamination [5]. For diagnosis of various diseases and increasing survivability rates early detection of abnormal physiological conditions is required. Microfluidics-MS is being used as a very competitive technology for biomarker discovery. The integration of LC microchip devices with MS detection, to screen biomolecular marker MCF-7 in breast cancer .The potential of an LC-ESI-MS chip for comparative proteomic analysis of isotopically labeled MCF-7 breast cancer cell extracts is explored for the first time. [6]. The threedimensional structures of proteins with a molecular weight up to $100 \mathrm{kDa}$, and solution structures of more than 100 plant proteins have been established by NMR spectroscopy. Highresolution NMR has become a key technology for the elucidation of biosynthetic pathways and metabolite flux via quantitative assessment of multiple isotopologues [7]. High-performance liquid chromatography has been developed for determination of salicylaldehyde isonicotinoyl hydrazone $(\mathrm{SIH})$ in rabbit plasma using $\mathrm{C} 18$ column This has provided the first information about the concentrations of $\mathrm{SIH}$ in plasma of a living subject. The results will have a significant impact on further progress in the development $\mathrm{SIH}[8]$.

\section{Chip and array technology}

These are solid-phase ligand binding assay systems using immobilised proteins on surfaces which include glass, membranes, microtiter wells, mass spectrometer plates, and beads or other particles. These assays are highly parallel and often miniaturized. Their advantages include being rapid and automatable, capable of high sensitivity, economical on reagents, and giving an abundance of data for a single experiment. Bioinformatics support is important; the data handling demands sophisticated software and data comparison analysis. It has applications in diagnostics for detection of antigens and antibodies in blood samples; profiling of sera to discover new disease markers; environment and food monitoring, protein expression profiling, protein-protein interactions; ligand-binding 
properties of receptors; enzyme activities [9]. Protein microarrays have been reportedly successful in serum tumor marker profiling and in drug discovery and medicinal chemistry. One of the emerging applications of protein microchips is biomarker discovery via chromatographic surface-based protein array techniques, which is applicable to minute amounts of samples with excellent detection limits using mass spectrometry based interrogation [13]. Protein chip array profiling analysis is potentially useful in monitoring the severity of disease in SARS patients A new strain of coronavirus (CoV), which had caused an outbreak of severe acute respiratory syndrome (SARS), the biomarkers were identified by protein chip array profiling using surface-enhanced laser desorption ionization time-of-flight mass spectrometry [10]. Three-dimensional cellular microarray has been developed for high-throughput toxicology assays where the Datachip consists of human cells encapsulated in collagen or alginate gels arrayed on a functionalized glass slide for spatially addressable screening against multiple compounds. A single DataChip containing 1,080 individual cell cultures, used in conjunction with the complementary human P450-containing microarray simultaneously provided IC50 values for nine compounds and their metabolites from CYP1A2, CYP2D6, and CYP3A4 and a mixture of the three P450s designed to emulate the human liver [11]. A peptide microarray to detect protein kinase activity in cell lysate has been developed. Where all the substrate peptides for kinases are immobilized chemoselectively on amino-coated glass slides and after phosphorylation of the immobilized peptides, it was detected by fluorescence imaging. This peptide microarray has found its usefulness in high-throughput kinase assay of intracellular signals and drug screening [12]. Nano-bio-chips has been used for high performance multiplexed protein detection where semiconductor nanoparticle quantum dots (QDs) has been integrated into a modular, microfluidic biosensor for quantitation of three important cancer markers, carcinoembryonic antigen (CEA), cancer antigen 125 (CA125), and Her-2/Neu (CerbB-2) has been achieved by using both serum and whole saliva specimens. Nano-bio-chips employs a fluorescence transduction signal with QD-labeled detecting antibody used in combination with antigen capture by a microporous agarose bead array supported within a microfluidics. This integrated nano-biochip assay system, in tandem with fluorophores, promises to be more sensitive tool for diagnostic and prognostic applications [14]. A novel stratergy of protein chip technology to screen agonists and antagonists of androgen receptor which is regarded as a significant drug targethas been developed which shows that he the $K(d)$ of testosterone and the $\mathrm{IC}(50)$ of $\mathrm{MT}$ (methyltestosterone) are consistent with the literature and 3 active ligands of $A R$ were identified with their IC( 50$)$ values of $3.63,2.19$, and 1.71 microM, respectively [16]. The electronic taste chip (ETC) assay system used for for the detection and measurement of interleukin (IL)-6. It offers a microchip platform on which bead-based immunoassays are performed. Each bead serves as its own independent self-contained "microreactor" system, with its selectivity determined by the specificity of the antibody that it hosts. This beadloaded chip is sandwiched between two optically transparent polymethylmethacrylate inserts, packaged within a metal casing, the flow cell. This flow cell allows for delivery of sample and detecting reagents to the microchip and the associated beads. Images of fluorescent beads are captured with a digital video chip and analyzed to facilitate detection and, ultimately, quantitation of analytes in complex fluids [16]. There is a chip-based method to detect protein glycosylation based on the energy transfer between quantum dots (QDs) and gold nanoparticles (AuNPs). The assay system relies on modulations in the energy transfer between the nanoparticles on a surface. The photoluminescence (PL) of lectin-coated QDs (energy donor) immobilized on a glass slide is quenched by carbohydrate-coated AuNPs (energy acceptor), and the presence of the glycoprotein causes the increase of the PL of QDs. Concanavalin A-coated QDs (ConA-QDs) and dextran-coated AuNPs (Dex-AuNPs) were used to detect the mannosylated proteins as a proof-of-concept. The PL intensity of QDs was found to be linearly correlated with the concentration and the number of glycan moiety of the glycoprotein. This assay system helps in analysis of glycoproteins with high selectivity and sensitivity in a high-throughput manner [17]. Microarray-based test are available for simultaneous and sensitive detection of biological warfare (BW) agents which include not only relevant viruses and bacteria, but also toxins. This application is not only restricted to the security and military sector but it can also be used in the fields of medical diagnostics or public health to detect, e.g., staphylococcal enterotoxins in food or clinical samples. The same technology could be used to detect antibodies against enterotoxins in human sera using a competitive assay. Protein and peptide microarrays can also be used for characterisation of antibodies. On one hand, peptide microarrays allow detailed epitope mapping. On the other hand, a set of different antibodies recognising the same antigen can be spotted as a microarray and labelled as detection antibodies. This approach makes it possible to test every combination, allowing finding the optimal pair of detection/capture 
antibody [18]. B cells are very heterogeneous, To identify single B cells with antigen specificity, cell microarray technology using microwell array chips has been developed where microwells, each capture a single $B$ cell. Monitoring antigeninduced intracellular $\mathrm{Ca} 2+$ mobilizations and the binding of fluorescence-labeled antigens simultaneously with a MAC-CCD system enables rapid detection of antigen-specific $B$ cells. This system provides a superior screening system for antigen-specific $B$ cells and extends the horizons of multiparameter single-cell analysis in heterogeneous cell populations [19].

\section{HPLC}

HPLC is a form of column chromatography technique used frequently in biochemistry and analytical chemistry to separate, identify, and quantify compounds. HPLC utilizes a column that holds chromatographic packing material (stationary phase), a pump that moves the mobile phase(s) through the column, and a detector that shows the retention times of the molecules. Retention time varies depending on the interactions between the stationary phase, the molecules being analyzed, and the solvent(s) used. There are various types of HPLC like Partition chromatography, Normal phase chromatography, Displacement chromatography, Reverse phase chromatography, Isocratic flow and gradient elution [20]. A universal HPLC detection technology, charged aerosol detection (CAD) is being used to overcome analytical problems for monitoring and measuring intact proteins, peptide, and amino acids. Charged aerosol detection (CAD) provides universal detection of proteins, peptides and amino acids. Utilization of CAD allows accurate and sensitive detection of these complex bimolecules by HPLC. With CAD, measuring biological therapeutics and even their formulation ingredients by HPLC has become routine. The combination of the attributes of CAD in a single HPLC detection platform has allowed the scientists in pharmaceutical development and manufacturing QC operations to replace older existing technologies [21]. RP-HPLC has been used for Purification and Molecular Identification of an Antifungal Peptide from the Hemolymph of Musca domestica (housefly). From the hemolymph an antifungal substance was isolated by solid-phase extraction combined with reverse phase-high performance liquid chromotography (RP-HPLC) and named as Musca domestica antifungal peptide-1 (MAF-1). Sodium dodecyl sulphate-polyacrylamide gel electrophoresis (SDS-PAGE) showed its molecular weight was $17 \mathrm{kDa}$. UV absorption spectra revealed that this antifungal substance possessed the characteristics of protein peptides. Edman degradation identified the sequence of 30 amino acids of its $\mathrm{N}$-terminal which matched no peptide in the MASCOT search database, indicating MAF-1 was a novel insect antifungal peptide. Mass spectrometry showed the precise Molecular weight of MAF-1 was 17203.384 Da. Its isoelectric point was acidic [22]. Multidimensional liquid chromatography, SCX-RP-LC as well as SAX-RP-LC, coupled to electrospray ion trap mass spectrometry has been used for the analysis of macromolecular protein complexes which is an important factor in understanding most cellular processes, e.g., protein transport into cell organelles, signal transduction via biological membranes, apoptosis, energy metabolism, directed motion of cells, and cell division. These complexes are not only built of various numbers of different proteins but also of prosthetic groups and RNA molecules [23]. Novel nonextractive and simple offline precolumn derivatization procedures have been proposed, for the trace determination of paroxetine (PXT) in human plasma by HPLC with fluorescence detection using Trimetazidine (TMZ) as an internal standard. The protein were derivatized with 7-chloro-4-nitrobenzo-2-oxa-1, 3-diazole and TMZ and PXT were separated using a Nucleosil $\mathrm{CN}$ column. The derivatized samples were excited at $470 \mathrm{~nm}$ and monitored at an emission wavelength of $530 \mathrm{~nm}$. This proposed method has high throughput, as the analysis involved a simple sample pretreatment procedure and short run time $(<10 \mathrm{~min})$. It showed that the method had great value when it is applied in the therapeutic monitoring of PXT [24]. There are implication that various lipoprotein classes are involved in the development of atherosclerotic cardiovascular disease has served to focus. One of the problems of such proteomic experimentation is the ability to initially isolate plasma lipoproteins subsequent to their analysis by mass spectrometry. Although there are several methods for the isolation of plasma lipoproteins available, they require large sample volumes and may cause destruction and dissociation of lipoprotein particle-associated proteins. Fast protein liquid chromatography (FPLC) is a non-denaturing technique which has been validated for the isolation of plasma lipoproteins from relatively small sample volumes. FPLC is used in conjunction with nanoHPLC-ESI-tandem mass spectrometry as a new integrated methodology suitable for the proteomic analysis of human lipoprotein fractions. It has been shown that only 200 microL of human plasma suffices for the isolation of whole HDL and the identification of the majority of all known HDL-associated proteins using mass spectrometry of the resulting fractions [25]. other application of HPLC is in pharmacokinetics and study of mitoxantrone in mice which is a simple, fast and an economical process [26]. 


\section{LC-MS}

The goal of many LC-MS proteomic investigations is to quantify and compare the abundance of proteins in complex biological mixtures. However, the output of an LC-MS experiment is not a list of proteins, but a list of quantified spectral features [27]. LC-MS is a powerful technique used for many applications, which has very high sensitivity and specificity. Generally, its application is oriented towards the specific detection and potential identification of chemicals in the presence of other chemicals (in a complex mixture) [21]. The use of performance enhancing substances are banned by WADA, the introduction of corticosteroids and designer drugs has made it essential to detect these critical doping agents it is done by using LC/MS/MS. A method was developed based on control $\mathrm{pH}$ extraction and HPLC/MS allowed better identification and separation of glucocortisteroid and few other drugs in different category from urine sample, this method has been used successfully for testing 1000 In-competition samples, it helps in selection of chemically and pharmaceutically banned drugs in urine in single short run at minimum required performance limit set by WADA [28]. The inner medullary collecting duct (IMCD) is an important site of vasopressinregulated water and urea transport. Mass spectrometry was used to investigate the proteome of the IMCD cell, and how it is altered in response to long-term vasopressin administration in rats. IMCD proteins were identified by liquid chromatography/ tandem mass spectrometry (LC-MS/MS) [29]. LC-MS has been used for identification of tyrosinephosphorylated proteins associated with metastasis and functional analysis of FER in human hepatocellular carcinoma cells using Hep3B (a nonmetastatic HCC cell line) and $\mathrm{MHCC} 97 \mathrm{H}$ (a highly metastatic HCC cell line). The tyrosine-phosphorylated proteins expressed in these cell lines were profiled by a phosphoproteomics technique based on LCMS/MS. [30]. A high throughput screening assay for the identification of ligands to pharmacologically significant receptors has been developed which is based on magnetic particles containing immobilized receptors followed by liquid chromatography-mass spectrometry (LCMS). The assay is suitable for the screening of complex mixtures such as botanical extracts. For proof-of-principle, estrogen receptor- $\alpha$ (ER- $\alpha)$ and ER- $\beta$ were immobilized on magnetic particles functionalized with aldehyde or carboxylic acid groups. Alternatively, biotinylated ER was immobilized onto streptavidin-derivatized magnetic particles. The ER that was immobilized using the streptavidin-biotin chemistry showed higher activity than that immobilized on aldehyde or carboxylic acid functionalized magnetic particles. Immobilized ER was incubated with extracts of Trifolium pratense L. (red clover) or Humulus lupulus L. (hops). As a control for nonspecific binding, each botanical extract was incubated with magnetic particles containing no ER. After magnetic separation of the particles containing bound ligands from the unbound components in the extract, the particles were washed, ligands were released using methanol, and then the ligands were identified using LCMS. The estrogens genistein and daidzein were identified in the red clover extract, and the estrogen 8-prenylnaringenin was identified in the hop extract [31]. LC-MS has future applications in metabolic profiling and metbolomic studies for biomarkers discovery. Emphasis is placed on recent developments in high efficiency LC separations, sensitive electrospray ionization approaches, and the benefits to incorporate both in LC-MS-based approaches [32]. Raltegravir is the first human immunodeficiency virus-1 (HIV-1) integrase inhibitor used in treatment-experienced patients who have evidence of viral replication and HIV-1 strains resistance to multiple antiretroviral regimens. Etravirine is a novel NNRTI, active against HIV-1 strains harboring multiple NNRTI mutations. A liquid chromatography-tandem mass spectrometry (LCMS/MS) method was developed for the quantification of raltegravir, etravirine, and 9 other antiretroviral agents (amprenavir, atazanavir, darunavir, efavirenz, indinavir, lopinavir, ritonavir, saquinavir, and tipranavir) in plasma at the concentrations associated with therapy which is rapid, specific, and sensitive method for routine therapeutic drug monitoring.[33]. Diverse taxa of cyanobacteria has been linked to biosynthesis of BMAA, a controversy had arisen about the detection of neurotoxic amino acids in cyanobacteria. A novel LC-MS/MS method was developed for the unambiguous determination of $\beta$ - $\mathrm{N}$-methylaminoI-alanine (BMAA) and 2,4-diaminobutyric acid (DAB) in cyanobacteria and selected plant seeds. Both neurotoxic and non-proteinogenic amino acids were analyzed without derivatization considering the total concentration of the free and protein-bound form. Overall 62 cyanobacterial samples worldwide revealed the absence of BMAA, whereas, the isomer DAB was confirmed in 16 cyanobacterial samples [34].

\section{Super Critical Fluid Chromatography}

SFC provides rapid separations without the use of organic solvents. It contributes no new chemicals to the environment. SFC separations can be done faster than HPLC separations because the diffusion of solutes in supercritical fluids is about ten times greater than that in liquids. Decrease in resistance to mass transfer in the column and allows for fast high resolution separations. Capillary SFC can provide highresolution chromatography at much lower 
temperatures. This allows fast analysis of thermo labile compounds [35]. Supercritical Fluid Chromatography is a form of normal phase chromatography that is used for the analysis and purification of low to moderate molecular weight, thermally labile molecules. It can also be used for the separation of chiral compounds. Principles are similar to those of high performance liquid chromatography (HPLC), however SFC typically utilizes carbon dioxide as the mobile phase; therefore, the entire chromatographic flow path must be pressurized [21]. Waste hops which are good sources of flavonoids were extracted using supercritical fluids technology [36]. Membrane proteins comprise $25-30 \%$ of the human genome and play critical roles in a wide variety of important biological processes. The detergents that are generally used to solubilize membrane proteins interfere with the crystallization process essential to X-ray studies and cause severe ion suppression effects that hinder mass spectrometric analysis. The use of supercritical fluid chromatography-mass spectrometry for the separation and analysis of integral membrane proteins and hydrophobic peptides shows that detergents are rapidly and effectively separated from the proteins and peptides, yielding them in a state suitable for direct mass spectrometric analysis [37]. A novel statergy for cancer theraphy is inhibition of the MDM2-p53 interaction can stabilize the p53 protein. The imidazoline compound (Nutlin-3) is a promising small molecule antagonist of the MDM2-p53 interaction. Various enantiomeric separation approaches has been exploredto resolve the Nutlin-3 enantiomer using chiral supercritical fluid chromatography (SFC) as well as chiral liquid chromatography (LC) under normal phase mode, reversed phase mode and polar organic phase mode.Using this $5 \mathrm{~g}$ of racemic mixture were purified on Prep-SFC in 75 min with the recovery rate above $92 \%$ [38]. N-Linked glycans have been characterized by supercritical fluid chromatography (SFC) and SFC-MS using positive- and negative-ion chemical ionization. The "soft" ionization conditions provide abundant molecular-weight-related anions for collisioninduced dissociation and subpicogram detection [39].

\section{Spectroscopic Analysis}

Spectrometry is the spectroscopic technique used to assess the concentration or amount of a given species. Spectroscopy/spectrometry is often used in physical and analytical chemistry for the identification of substances through the spectrum emitted from or absorbed by them. The type of spectroscopy depends on the physical quantity measured. Normally, the quantity that is measured is intensity, either of energy absorbed or produced [21]. Information on the structure and functional organization of membrane proteins is lacking in spite of their functional importance due to paucity of crystal structures. In the absence of a detailed crystallographic database, approaches based on fluorescence spectroscopy have proved to be useful in elucidating the organization, topology and orientation of membrane proteins. A particular advantage of approaches based on fluorescence spectroscopy is the multiplicity of measurable parameters which complement each other in terms of their information content. In cases where an external fluorophore is used, there is a choice of the fluorescent label to be used, and therefore, specific probes with appropriate characteristics can be designed for specific applications. The lack of precise crystallographic resolution is often compensated by the dynamic nature of the information obtained when fluorescence-based approaches [40]. In protein-folding studies it is often required to differentiate a system with only two-states, namely the native $(\mathrm{N})$ and unfolded (U) forms of the protein present at any condition of the solvent, from a situation wherein intermediate state(s) could also be present. Time-resolved fluorescence has the capability to reveal the presence of heterogeneity of structural forms due to the'fingerprint' nature of fluorescence lifetimes of various forms i.e., quantitative analysis of amplitudes associated with fluorescence lifetimes in multiexponential decays. The relative population of species from two-component mixtures of non-interacting molecules such as fluorescent dyes, peptides and proteins can be estimated accurately. The amplitudes of fluorescence lifetimes are controlled by fluorescence resonance energy transfer (FRET), that the equilibrium foldingunfolding transition of the small singledomain [41]. Deep UV resonance Raman (DUVRR) spectroscopy combined with hydrogendeuterium exchange and advanced statistical analysis, 2D correlation in particular, allow for quantitative characterization of protein structural evolution at all stages of fibrillation in vitro. Amyloid fibrils associated with numerous degenerative diseases are non-crystalline and insoluble, and thus are not amenable to conventional X-ray crystallography and solution NMR, is studied by DUVRR [42]. The 37-residue human islet amyloid polypeptide (hIAPP or amylin) self-assembles into fibers, the assembly of which has been associated with the disease mechanism of type II diabetes. Infrared spectroscopy in conjunction with isotope labeling has proved to be a powerful tool for studying the aggregation process of hIAPP and other amyloid forming proteins with residue specific structure and kinetic information, 2D IR spectra distinguishes between residues in beta-sheets versus those in turn regions, and that transitional residues between secondary structures can be identified by the suppression of their cross-peaks 
in 2D IR spectra. It is not limited to amyloid fibrils is generally useful in identifying regions of secondary structure in proteins using 2D IR spectroscopy and isotope labeling [43]. Because of its capacity to monitor multiple biomolecular parameters, Fourier transform infrared( FTIR) spectroscopy enables unambiguous and easy analysis of cell death modes and may be useful for biochemical and medical applications [44]. Raman spectroscopy has been recognized as a valuable analytical tool in biological and medical research. This technique allows probing molecular vibrations of samples without external labels or extensive preparation. This nondestructive optical technique can provide rapid and objective and reproducible measurements of sample biochemistry and identify variations that occur between healthy and diseased tissues. In fact, biochemical changes within tissue may either initiate disease or occur as a result of the disease process. The qualitative analysis of such changes provides important clues in the search for a specific diagnosis and the quantitative analysis of biochemical abnormalities is important in measuring the extent of the disease process, designing therapy and evaluating the efficacy of treatment. Near-infrared Raman microspectroscopic imaging has been used as a diagnostic tool to investigate, ex vivo, the changes between normal and adenocarcinomatous human colonic tissues. [45].Recently it has been shown that the same individual protein and its photoproducts can be detected via Fluorescence and SurfaceEnhanced Raman Spectroscopic Imaging [46]. By using three different methods, extracellular polymeric substances (EPS) were extracted from the bacterial strain Pseudomonas putida and the fungal species Aureobasidium pullulans. The composition of EPS was analyzed by biochemical and high-resolution solid state 13C nuclear magnetic resonance (NMR) spectroscopic methods. The NMR method showed that about $40 \%$ of the EDTA accumulated in the EPS and that was responsible for the apparent high extraction yields when using EDTA extraction. EPS protein content determined by the NMR method was up to $30 \%$ higher than the protein content determined using the biochemical (Lowry) method for P. putida and for A. pullulans [47]. Detection of peripheral zone prostate cancer in patients with elevated levels prostate specific antigen and low free-to-total PSA ratio is now-adays being carried out using MR imaging and MR spectroscopy [48].

\section{GMP compliance}

Good manufacturing practice (GMP) is that part of quality assurance which ensures that products are consistently produced and controlled to the quality standards appropriate to their intended use and as required by the marketing authorization. GMP is aimed primarily at diminishing the risks inherent in any pharmaceutical production, which may broadly be categorized in two groups: cross contamination/mix-ups and false labelling. Above all, manufacturers must not place patients at risk due to inadequate safety, quality or efficacy; for this reason, risk assessment has come to play an important role in WHO quality assurance guidelines [53].The Food and Drug Administration (FDA) mandates that a drug firm, and therefore its laboratory, be operated in a state of control by employing conditions and practices that assure compliance with the intent of The Federal Food, Drug, and Cosmetic Act and portions of the Current Good Manufacturing Practice (CGMP) regulations that pertain to it [49]. FDA considers a firm's laboratory control system to be a key element in CGMP compliance. Within the laboratory control systems are at least seven additional subsystems or sub elements which include:

- Laboratory managerial and administrative systems,

- Laboratory documentation practices and standard operating procedures,

- Laboratory equipment qualification and calibration,

- Laboratory facilities,

- Methods validation and technology transfer,

- Laboratory computer systems, and

- Laboratory investigations. [49]

The GMP compliant companies are large pharmaceutical companies with many employees in production, testing, support and administrative having equal or outnumber $R$ \& $D$ scientists who follow accepted, validated, approved methods and having large volume of documentation [50]. A company moves from a pre-GMP to GMP when company has identified product to produce; plan needed to develop product under GMP, be part of company master plan site able to produce GMP product or must find alternate manufacturing facility, regulatory affairs personnel - always jobs in this area, finalize the plan, determine budget (may be when a company goes public) START the project and documentation [50]. Documentation is important in order to show control, data is needed to support your position. These data are obtained by executing a well-organized and systematic laboratory audit. The goals of a laboratory audit are:

Demonstrate control by conducting the audit and generating data to support your position.

If not in control then:

Show that you know why you are not in control;

- Show that you know which areas are out of compliance;

Show that you know which areas have the greatest impact; 
Develop interim controls to mitigate the impact

of the areas with the greatest risk;

Develop a plan to put you back in control; [49]

The GMP guidelines for biological products have been approved by both the WHO Expert Committee on Biological Standardization and the WHO Expert Committee on Specifications for Pharmaceutical Preparations. The specialized GMP guidelines for the manufacture of herbal medicinal products address the manufacture of products from material of plant origin, which may be subject to contamination and deterioration and vary in its composition and properties [51]. A strategy has been validated for endotoxin, sterility and potency testing in an advanced. Therapy medicinal products (ATMP) used in cardiac regeneration. As new ATMPs are developed, the regulatory frameworks have evolved and the existing regulations provide an appropriate structure for ensuring the safety and efficacy of the next generation of ATMPs. Personnel have to be adequately trained on relevant methods and their application to stem-cell-based products [54]. Biodrugs (biologics) is much more complex than chemically synthesized drugs because of their structural heterogeneity and interactions within a given biologic system. The manufacturing process in the biodrug industry varies with each type of molecule and is far more elaborate and stringent due to the use of living organisms and complex substrates. Product purity and altered structural characteristics leading to potential immunogenicity have often been of concern when establishing quality and safety in the use of biodrugs. Regulatory compliance in manufacturing and commercialization of biodrugs involves quality control, quality assurance, and batch documentation. Good Manufacturing Practice (GMP) for biodrugs has been developed in certain regions such as the EU, US, and Japan. There are variations in GMP guidelines between countries, which lead to difficulty for the manufacturers in conforming to different standards, thus entailing delays in the commercialization of biodrugs. There is a need to develop a unified regulatory guideline for biodrug manufacturing across various countries, which would be helpful in the marketing of products and trade. This review deals with the comparative framework and analysis of GMP regulation of biodrugs [55]. Intracoronary application of BMderived cells for the treatment of acute myocardial infarction (AMI) is currently being studied intensively. There are strict legal requirements that surround the production of cells for clinical studies. Thus good manufacturing practice (GMP)-compliant collection and preparation of BM for patients with AMI was established by the Cytonet group. The increased expense of the GMP-compliant process can be justified by higher safety for patients and better control of the final product [56].

\section{Tools and Softwares for Protein Analysis}

The National Center for Biotechnology Information (NCBI) at the National Institutes of Health was created in 1988 to develop information systems for molecular biology. In addition to maintaining the GenBank nucleic acid sequence database, which receives data through the international collaboration with DDBJ and EMBL as well as from the scientific community, $\mathrm{NCBI}$ provides data retrieval systems and computational resources for the analysis of GenBank data and many other kinds of other biological data. NCBI resources include Entrez, the Entrez Programming Utilities, MyNCBI, PubMed, PubMed Central, Entrez Gene, the NCBI Taxonomy Browser, BLAST, BLAST Link (BLink), Electronic PCR, OrfFinder, Spidey, Splign, Reference Sequence, UniGene, HomoloGene, ProtEST, dbMHC, dbSNP, Cancer Chromosomes, Entrez Genomes and related tools, the Map Viewer, Model Maker, Evidence Viewer, Trace Archive, Sequence Read Archive, Retroviral Genotyping Tools, HIV-1/Human Protein Interaction Database, Gene Expression Omnibus, Entrez Probe, GENSAT, Online Mendelian Inheritance in Man, Online Mendelian Inheritance in Animals, the Molecular Modeling Database, the Conserved Domain Database, the Conserved Domain Architecture Retrieval Tool, Biosystems, Peptidome, Protein Clusters and the PubChem suite of small molecule databases. Augmenting many of the web applications are custom implementations of the BLAST program optimized to search specialized data sets.

\section{Recent Developments}

Biosystems is a new database within Entrez that collects together molecules that interact in a biological system, such as a biochemical pathway or disease.

\section{BLAST improvements and updates}

There have been three main improvements to the NCBI BLAST web site this year. The first is the addition of Sequence Read Archive (SRA) transcript libraries as a new search set, which includes all public sequences from 454 sequencing systems. These sequences can be searched using the 'Search SRA transcript libraries' link in the 'Specialized BLAST' section of the BLAST web site. NCBI has also reorganized the page for aligning two sequences using BLAST (bl2seq), which now has a search page consistent with the other BLAST pages. Finally, the BLASTP report now offers a new 'Multiple Alignment' option that uses COBALT to perform a multiple alignment of the query sequence and any subject sequences listed in the BLAST report. If the user selects this link, a 
separate multiple alignment search is started and displayed in a separate browser window.

\section{COBALT}

COBALT is a new multiple alignment algorithms, which finds a collection of pairwise constraints derived from both the NCBI Conserved Domain database (CDD) and the sequence similarity programs RPS-BLAST, BLASTP and PHIBLAST.

\section{Peptidome}

Peptidome is a new data repository for tandem mass spectrometry peptide and protein identification data generated by the scientific community [58]. Sequence analysis encompasses the use of various bioinformatics methods to determine the biological function and/or structure of genes and the proteins they code for.

\section{Conclusion}

This review paper highlights on various equipments and instruments used in proteomics or proteins analysis and also provides information on various modifications made in instrumentation for better analysis of proteins .the article also provide latest application of various validations and modifications in instrumentation. Various tools and software's for the protein analysis are also mentioned along with the GMP compliances which one should follow under the guidelines of US-FDA and WHO.

\section{References}

[1] Pace Silvia, Capocasa Francesca, Tallarico Carlo, Frapolli Roberta, Zucchetti Massimo, Longo Antonio. (2009) Journal of pharmaceutical and biomedical analysis 50(3):507-14.

[2] Sala Federica, Zucchetti Massimo, Bagnati Renzo, D'Incalci Maurizio, Pace Silvia, Capocasa Francesca, Marangon Elena. (2009) Journal of chromatography. B, Analytical technologies in the biomedical and life sciences, 877(27):3118-26.

[3] Feng K., Wang S., Hu D.J., Yang F.Q., Wang H.X., Li S.P. (2009) Journal of pharmaceutical and biomedical analysis, 50(3):522-6.

[4] Orbán Norbert, Kozák Imre Olivér, Drávucz Mónika, Kiss Attila. (2009) International Journal of Food Science \& Technology, 44(4), 869-873.

[5] Becker J.S., Zoriy M., Matusch A., Wu B., Salber D., Palm C., Becker J.S. (2009) Mass Spectrom Rev., [PMID: 19557838]

[6] Armenta J.M., Hoeschele I., Lazar I.M. (2009) Journal of the American Society for Mass Spectrometry 20(7): 12871302.
[7] Eisenreich W. and Bacher A. (2007) Photochemistry, 2799-2815.

[8] Kovaríková P., Klimes J., Stĕrba M., Popelová O., Mokrý M., Gersl V., Ponka P. (2005) Journal of Separation Sciences, 2799-2815.

[9] UniProt Consortium. (2009) Nucleic Acids Res., 37(Database issue):D169-74.

[10] Yip T.T., Chan J.W., Cho W.C., Yip T.T., Wang Z., Kwan T.L., Law S.C., Tsang D.N., Chan J.K., Lee K.C., Cheng W.W., Ma V.W., Yip C., Lim C.K., Ngan R.K., Au J.S., Chan A., Lim W.W. (2005) Clinical Chemistry, 47-55.

[11] Lee M.Y., Kumar R.A, Sukumaran S.M., Hogg M.G., Clark D.S., and Dordick J.S. (2007) Proceedings of the National Academy of Sciences of USA, 59-63.

[12] Shigaki S., Yamaji T., Yamanouchil X., Sonoda T., Okitsu O., Moril T., Niidome T. and Katayama Y. (2007) Analytical Sciences, 271.

[13] Spisák S., Guttman A. (2009) Current Medicinal Chemistry, 2806-2815.

[14] Jokerst J.V., Raamanathan A., Christodoulides N., Floriano P.N., Pollard A.A , Simmons G.W., Wong J., Carole Gage, Furmaga W.B., Redding S.W. and McDevitt J.T. (2009) Biosensors and Electronics, 3622-3629.

[15] Zhou Y., Liu A., Wang W., Du G. (2008) Journal of Biomolecular Screening, 276.

[16] Christodoulides N., Dharshan P., Wong J., Floriano P.N., Neikirk D., McDevitt J.T.( 2007) Methods in Molecular Biology, 131-44.

[17] Kim Y.P., Park S., Oh E., Oh Y. and Kim H. (2009) Biosensors and Bioelectronics, 1189-1194.

[18] Ehricht R., Adelhelm K., Monecke S., Huelseweh B. (2009) Methods in molecular Biology, 85-105.

[19] Kinoshita K., Ozawa T., Tajiri K., Kadowaki S., Kishi H., Muraguchi A. (2009) Cytometry part A:the journal of the international society for Analytical Cytology. $682-687$.

[20] Rieux L., Lubda D., Niederländer H.A., Verpoorte E., Bischoff R. (2006) J Chromatogr A., 1120(1-2):165-72.

[21] Sarmento B., Ribeiro A., Veiga F., Ferreira D. (2006) Biomed Chromatogr., 20(9):898-903.

[22] Fu P., Wu J. and Guo G. (2009) Cellular \& Molecular Immunology, 245-51.

[23] Wagner Y., Sickmann A., Meyer H.E., Daum G. (2003) Journal of the American Society for Mass Spectrometry, 1003-1011.

[24] Darwish I.A., Al-Majed A.A., Mahmoud A.M., Khalil N.Y. (2009) J AOAC Int., 92(5):1349-55 
[25] Collins L.A., Mirza S.P., Kissebah A.H., Olivier M. (2009) Physiological Genomics, [PMID: 19903763].

[26] An G. and Morris M.E. (2009) Journal of Pharmaceuticals and Biomedical analysis, 51(3):750-3.

[27] Clough T., Key M., Ott I., Ragg S., Schadow G., Vitek O. (2009) Journal of proteome research, 5275-5284.

[28] Reddy I.M., Beotra A., Jain S., Ahi S. (2009) Indian Journal of Pharmacology, 80-86.

[29] Pisitkun T., Bieniek J., Tchapyjnikov D., Wang G., Wu W.W., Shen R.F., Knepper M.A. (2006) Physiological Genomics, 25(2):263-76.

[30] Li H., Ren Z., Kang X., Zhang L., Li X., Wang Y., Xue T., Shen Y. and Liu Y. (2009) BMC Cancer, 9:366.

[31] Choi Y. and van Breemen Richard B. (2008) Combinatorial chemistry and High throughput screening, 11(1):1-6.

[32] Metz T.O., Zhang Q., Page J.S., Shen Y., Stephen J. Callister, Jon M. (2007) Biomarkers in Medicine, 159-185.

[33] Quaranta S., Woloch C., Paccou A., Giocanti M., Solas C., Lacarelle B. (2009) Theraupetic Drug Monitoring, 31(6):695-702.

[34] Krüger T., Mönch B., Oppenhäuser S. and Luckas B. (2009) Toxicon., [PMID: 19825383].

[35] Ficetola G.F., Miaud C., Pompanon F., Taberlet P. (2008) Biol Lett., 4(4):423-5.

[36] He U., Xiong H., Chen Q., Ruan H., Wang Z. and Traore L. (2005) PMC Journal. pg no. 999-1004.

[37] Zhang X., Scalf M., Westphall M.S., Smith L.M. (2008) Anal Chem., 80(7):2590-8.

[38] Wang Z, Jonca M, Lambros T, Ferguson S, Goodnow R. (2007) J Pharm Biomed Anal., 45(5):720-9.

[39] Sheeley D.M., Reinhold V.N. (1998) Anal Chem., 70(14):3053-9.

[40] Chattopadhyay A. and Raghuraman $\mathrm{H}$. (2004) Current Sciences, 175-180.

[41] Saxena A.M., Krishnamoorthy G., Udgaonkar J.B. and Periasamy N. (2007) Journal of Chemical Sciences, 61-69

[42] Lednev I.K., Shashilov V. and Xu M. (2009) Special Section: Raman Spectroscopy Current Science, 80-185.

[43] Strasfeld D.B., Ling Y.L., Gupta R., Raleigh D.P., Zanni M.T. (2009) J Phys Chem B., 113(47):15679-91.

[44] Zelig U., Kapelushnik J., Moreh R., Mordechai S., Nathan I. ( 2009) Biophysical Journal, 97(7):2107-14.

[45] Beljebbar A., Bouché O., Diébold M.D., Guillou P.J., Palot J.P., Eudes D., Manfait M. (2009) Critical Reviews in Oncology/Hematology, 255-264.
[46] Schleifenbaum F., Blum C., Elgass K., Peter S., Subramaniam V., Meixner A. (2009) J. Proc. SPIE $7190,71900 U$.

[47] Metzger U., Lankes U., Fischpera K., Frimmel F.H. (2009) Applied Microbiology and Biotechnology,197206.

[48] Vilanova J.C., Comet J., Barceló-Vidal C., Barceló J., López-Bonet E., Maroto A., Arzoz M., Moreno A., Areal J. (2009) Radiology, 253(1):135-43.

[49] David M. Bliesner (2006) Establishing $A$ CGMP Laboratory Audit System: $A$ Practical Guide, ISBN: 978-0-47173840-4, John Wiley \& Sons, Inc.

[50] Sidney Willig (2000) Good Manufacturing Practices for Pharmaceuticals: A Plan for Total Quality Control from Manufacturer (Drugs and the Pharmaceutical Sciences: a Series of Textbooks and Monographs), ISBN-13: 978-0824704254.

[51] World Health Organization (2004) Quality Assurance of Pharmaceuticals, Vol. 2, ISBN-13: 978-9241546195.

[52] Richard Guarino (2004) New Drug Approval Process, Fourth Edition, Accelerating Global Registrations (Drugs and the Pharmaceutical Sciences), Informa HealthCare, ISBN-13: 978-0824750411.

[53] Gian Luca Burci and Claude-Henri Vignes (2004) World Health Organization, Kluwer Law International, ISBN-13: 9789041122735.

[54] Soncin S., Cicero VL, Astori G., Soldati G., Gola M., Sürder D. and Moccetti T. (2009) Journal of Translational Medicine, 7:78.

[55] Sahoo N., Choudhury K., Manchikanti P. (2009) Biodrugs: clinical immunotherapeutics biopharmaceuticals and gene therapy, 217-229.

[56] Griesel C., Heuft H.G., Herrmann D., Franke A., Ladas D., Stiehler N., Stucki A., Braun M., Wollert K.C., Meyer G.P., Drexler H., Hertenstein B., Ganser A., Rüdinger W., Arseniev L. (2007) Cytotherapy, 35 - 43

[57] Paul F. Basch (1999) Textbook of International Health, Oxford University Press, USA, ISBN-13: 978-0195132045.

[58] Sayers E.W., Barrett T., Benson D.A., Bolton E., Bryant S.H., Canese K., Chetvernin V., Church D.M., Dicuccio M., Federhen S., Feolo M., Geer L.Y., Helmberg W., Kapustin Y., Landsman D., Lipman D.J., Lu Z., Madden T.L., Madej T., Maglott D.R., Marchler-Bauer A., Miller V., Mizrachi I., Ostell J., Panchenko A., Pruitt K.D., Schuler G.D., Sequeira E., Sherry S.T., Shumway M., Sirotkin K., Slotta D., Souvorov A., 
Starchenko G., Tatusova T.A., Wagner L., Wang Y., John Wilbur W., Yaschenko E., Ye J. ( 2009) Nucleic Acid Res., [PMID: 19910364].

[59] McWilliam H., Valentin F., Goujon M., Li W., Narayanasamy M., Martin J., Miyar T., Lopez R. (2009) Nucleic Acids Res. 37(Web Server issue):W6-10. 